\section{Practical SPECT/CT in Nuclear Medicine}

D. W. Jones, P. Hogg, and E. Seeram, Eds.

New York, NY: Springer, 2013, 345 pages, $\$ 79.99$

Today SPECT/CT has become standard in many imaging pathways, and thus there is a great need for information on how to achieve optimal SPECT/CT images. SPECT/CT can provide unique information that would otherwise be unavailable if SPECT and CT were used in isolation from each other. This unique information can assist in important decision making about patient diagnosis and management. The technologic advances made for PET/CT have no doubt advantaged the progression of SPECT/CT technology and perhaps influenced clinicians in their purchasing decisions in relation to hybrid imaging more generally.

Although intended primarily for radiographers and nuclear medicine technologists who work with SPECT/CT, this book is also valuable for other professionals within medical imaging. Practical insight is given into SPECT/CT, some of its uses are outlined, and how to perform some of the more common procedures is explained. For nuclear medicine professionals, a fairly large amount of material on or related to CT has been included, but a tremendous amount of information on nuclear medicine has purposely not been included.

The book is divided into 5 parts and 13 chapters written by 16 experts, mostly from the United Kingdom. Part I, with 5 chapters, was written by physicists and diagnostic radiographers and considers the physics and equipment for the operation of a SPECT/CT system. Chapters 2 and 3 discuss basic electromagnetic radiation, beam characteristics, CT image quality, and radiation dose to the patient. Chapter 4 deals with basic $\gamma$-camera SPECT, and chapter 5 is dedicated to CT instrumentation and quality control. Part II, with 2 chapters, considers practical information needed to implement a SPECT/CT system into a clinic. Chapter 6 elaborates on considerations in choosing a system, and chapter 7 advises on facility and room designs for housing a SPECT/CT system. Part III, with 1 chapter, addresses medical uses of SPECT/CT and includes many SPECT/CT cases and images. Part IV has 2 chapters. Chapter 9 deals with radiation protection within the hybrid SPECT/CT environment, and chapter 10 covers optimization of $\mathrm{CT}$ acquisition parameters. Part V, with 3 chapters, outlines a practical perspective on using SPECT/CT. Chapter 11 reviews cross-sectional anatomy for CT positioning purposes, and chapter 12 considers radiographic contrast media and injection systems used in CT. The final chapter, 13, outlines a range of common SPECT/CT procedures with suggested protocols for achieving a successful scan.

This book is a practical guide to SPECT/CT imaging with good illustrations. The references are updated, and the index is helpful. I highly recommend this book to trainees and

COPYRIGHT @ 2013 by the Society of Nuclear Medicine and Molecular Imaging, Inc. practitioners in nuclear medicine, radiology, and medical physics.

\author{
E. Edmund Kim \\ University of California at Irvine \\ 101 The City Dr. S. \\ Orange, $C A 92868$ \\ E-mail: edmundek@uci.edu
}

Published online Sep. 26, 2013.

DOI: 10.2967/jnumed.113.132209

\section{Atlas of Nuclear Cardiology}

V. Dilsizian and J. Narula, Eds.

New York, NY: Springer, 2013, 457 pages, \$209

Nuclear cardiology imaging is solidly based on many branches of science and engineering, and the strengths of nuclear techniques are related to physiologic, biochemical, and molecular properties. The ability to define myocardial perfusion, viability, and ventricular function from a single study has become a powerful diagnostic and prognostic tool, and thus nuclear cardiology is now recognized as a distinct clinical entity. Nuclear cardiology procedures have become the cornerstone of the decision-making process to appropriately select patients for medical or interventional therapy, as well as to monitor the effectiveness of that therapy. Innovative imaging strategies in nuclear cardiology will propel the field into molecular imaging and personalized medicine.

The object of this fourth edition of the Atlas of Nuclear Cardiology is to elucidate the role of cardiovascular nuclear procedures in the clinical practice of cardiology. By allowing quantification of blood flow, PET has led to a better understanding of the physiologic mechanism underlying cardiovascular diseases. Targeted molecular imaging and image-guided therapy further improve the management of heart diseases. In this atlas, diagnostic algorithms and schematic diagrams integrated with nuclear cardiology procedures are generally interspersed with superb color figures and tables to emphasize key concepts in cardiovascular physiology, pathology, metabolism, and innervation. References are updated, and the index is convenient.

The atlas consists of 12 chapters written by 27 international experts. The first chapter presents the principles of nuclear cardiology imaging along with instrumentation and image acquisition. Chapters 2-4 detail SPECT and PET radiopharmaceuticals, imaging techniques, and stressors for the detection of coronary artery disease. Chapter 5 deals with the benefits of quantification of myocardial perfusion, and chapter 6 reviews techniques for radionuclide angiography and myocardial perfu- 